\title{
Philosophiques
}

\section{Dialectique de la communication chez Kierkegaard}

\section{Jacques Caron}

Volume 3, numéro 2, octobre 1976

URI : https://id.erudit.org/iderudit/203052ar

DOI : https://doi.org/10.7202/203052ar

Aller au sommaire du numéro

Éditeur(s)

Société de philosophie du Québec

ISSN

0316-2923 (imprimé)

1492-1391 (numérique)

Découvrir la revue

Citer cet article

Caron, J. (1976). Dialectique de la communication chez Kierkegaard.

Philosophiques, 3(2), 167-181. https://doi.org/10.7202/203052ar d'utilisation que vous pouvez consulter en ligne.

https://apropos.erudit.org/fr/usagers/politique-dutilisation/ 


\title{
DIALECTIQUE DE LA COMMUNICATION CHEZ KIERKEGAARD
}

\author{
par Jacques Caron
}

La dissimulation cœxiste avec l'expression. Dans l'œuvre de Kierkegaard, les prisons prolifèrent. Accomplir la généralité (det Almene), c'est-à-dire l'éthique, est une prison ; le pastorat éventuel est une prison ; le mariage est une prison ... Tour à tour, elles surgissent, ombres menaçantes, des textes de Johannès de Silentio et de Vigilius Haufniensis sous les noms de repliement-sur-soi, de non-liberté, de non-communication.

Comment alors se délier de ces prisons puisque le récit doit déchirer le silence et constituer la " geste " du langage ? La parole est réverbération du sens du monde ; elle est naissance répétée et infinie et par cela même le contraire de la mort et de l'angoisse. En effet, l'être parlant est déplié, ouvert, continu, libre (voir à ce propos les analyses du Concept de l'Angoisse) : par la communication, le moi lié pourra s'exprimer. L'angoisse est l'approche la, plus vertigineuse du désir de communiquer ; à partir d'une position bouleversée, elle vient à elle, se forme en formant, est tentée jusqu'à la folie et tente jusqu'au possible, au possible de la liberté.

D'autre part, si Johannès de Silentio et Vigilius Haufniensis (la question se pose aussi pour Anti-Climacus de la Maladie à la Mort) expriment le repliement, ils expriment en même temps l'exception, l'hétérogénéité, l'uensartet (le "dissemblable ").

Impossibilité donc pour ces auteurs de réaliser la généralité, mais, par contre, ils s'aménagent un site supérieur qui sauve de la suspension non seulement éthique mais existentielle: l'histoire humaine dilue l'histoire du sens.

Nous reconnaissons, en effet, la souplesse avec laquelle Kierkegaard se prend à ses masques; alors, si nous réfléchissons sur la dialectique du repliement et de l'exception, on peut se poser la question suivante : existe-t-il une communication ou un semblant 
de communication entre un repliement (qui est "forcé ») et une exception (qui est "admise ") ? Nous croyons comprendre qu' une comparaison de sens est possible entre le replié et l'exception (en vérité, ils sont tous deux des isolés: Abraham et S $\phi$ ren Kierkegaard) ; le replié a la possibilité de communiquer, mais incognito, tandis que l'exception s'identifie à une mission (l'exception de l'éthique se métamorphose en chargé de mission, en Extraordinaire, en Martyre, en Génie etc . . sous la dépendance directe d'une puissance supérieure, d'un gouvernement divin, de la providence) de plus en plus précise, mais dont la signification demeure en grande partie hermétique pour la majorité des "lecteurs" (pensons aux contemporains de Kierkegaard par exemple). La délivrance du discours se fera attirante au possible et servira le message religieux.

L'un replié à cause des "hommes", l'autre à cause d'une puissance supérieure, ils infléchissent une image fictive qui s'interpose entre la réalité du monde et la réalité supérieure : il y aura communication, mais une communication préservant le caractère de la fiction, une communication indirecte, une communication incognito qui laisse à "det Enkelte " suffisamment de possibilités pour se réaliser en fonction de la communication religieuse. Le vouloir-dire s'entend très bien en pseudonymie, mais en pseudonymie seulement; Kierkegaard a horreur de l'anonymat qui annihile tout élément personnel dans un discours, qui rapporte la communication (contenu) à quelque chose d'extrêmement objectif. Ainsi, le pseudonyme préserve l'aspect réalisateur du moi et tout effort de réalisation se ramène d'emblée à reconnaître le médium de la communication indirecte comme la seule communication.

À partir de ce point, et que ce soit pour le poète ou pour le martyre, la liberté est une conquête : le devoir de s'ouvrir, même et surtout en usant de la pseudonymie ou du masque, n'a jamais été contesté.

"Tout mon être est changé ", écrit Kierkegaard. "Mon repliement et ma taciturnité sont brisés, je dois parler. "'

1. L'édition du "Journal" est la suivante: Papirer, udg., af P.-A. Heiberg, V. Kuhr, E. Torsting, Kфbenhavn, $1909 \mathrm{ff}$. (I-XI). Toutes nos citations sont tirées de cette édition.

Pap. VIII, 1, 640.

Cette note du 19 avril (1848). Le 24 avril, Kierkegaard écrit : "Non, non, mon re- 
La mise en service de Kierkegaard exige que soit niée l'incarcération du sens, c'est-à-dire que son expérience soit opposée au système (le langage formel). C'est en ce sens que nous donnons ici un bref aperçu de la théorie kierkegaardienne de la communication ("Den ethiske og den ethisk-religieuse Meddelelses dialektik, " 1847) ${ }^{2}$.

Quelle était l'intention de Kierkegaard en rédigeant ces notes pour une théorie de la communication? Vers 1846-7-8, Kierkegaard sentait (ses notes en font foi) la nécessité de "penser " son œuvre antérieure, du moins d'y jeter un regard rétrospectif qui soit en même temps explicatif. Point de vue explicatif de mon cuure marque le premier jalon sur cette voie. Le but que Kierkegaard se propose est d'expérimenter sur le fait le fonctionnement d'une communication directe et indirecte et d'établir le caractère "maïeutique", formateur, réalisateur de toute communication ayant pour sujet un élément esthétique-éthique et esthético-éthicoreligieux. L'importance est particulièrement donnée à l'éthique et à l'éthico-religieux : elle marque le rapprochement unitaire des affluents et des confluents de la communication indirecte (indirekte Meddelelsen) qui tient pour thématique centrale la circonscription et l'englobement de l'explication-formation. D'autre part, l'esthétique et l'éthique forment surtout des obstacles extérieurs alors que le religieux, dans son avenir communicatif, a possibilité de s'attacher une certitude de connaissance, du moins au niveau de la religiosité-A. (Communication de savoir (Viden) provisoire (Forl $\phi$ bige), qui exige une communication directe (direkte Meddelelse). Mais c'est là un " cas " exceptionnel. Car, pour parler directement, il faut se bien posséder : nulle dissimulation, nulle exception, mais la permanence d'une parole qui est en même temps pétrification.

Kierkegaard commence par constater la "sécheresse ", l'inhumanité de la connaissance moderne. Il va en démontrer l'essence

pliement ne se laisse pas rompre, tout au moins pas maintenant" (Pap, VIII, A, 645). L'oscillation est constante entre repliement et ouverture. Voir les notes sur la mélancolie (repliement), vidage du moi dans l'œuvre: Pap. VIII A, 27.44.87.

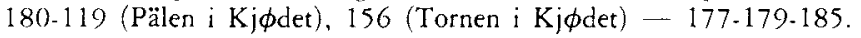

L'expérience doit être opposée au système - le langage - et prendre la place de ce dernier. C'est dans ce sens que nous donnons un aperçu de la théorie de la communication kierkegaardienne.

2. La traduction que nous en donnons n'est pas complète; il s'agit d'extraits, mais inédits en français. 
malbonnête (Uredelig) : "Si l'on devait caractériser par un seul mot la connaissance moderne - ou mieux, l'erreur de l'époque moderne, surtout depuis qu'on a quitté la voie royale de Kant et donné les cent rixdales pour devenir théocentrique - alors, on devrait dire : elle est malhonnête. malhonnêteté manque de naïveté $n^{3}$.

Cette malhonnêteté moderne, rien ne la cache : elle se manifeste sous nos yeux, à tous les jours, sous d'innombrables, d'inlassables, d'innommables façons, par exemple dans l'illusion de soi-même (Selvbedrag), dans la confusion (Vilderede). De plus, cette malhonnêteté est une réplique du manque de naïveté : "le naîf et l'acquis. Ce n'est pas un signe de maturité que d'avoir cessé d'être naîf ou de ne jamais l'avoir été. La naïveté doit être conservée - à trancher entre ce qu'on comprend et ce qu'on ne comprend pas - prédominance de la génération. Une génération est une concrétion abstraite et ne peut jamais être naïve ${ }^{4}$. La connaissance, poursuit Kierkegaard, "est devenue imaginative (savoir pur) - - en outre, toujours apprise - les combinaisons ridicules "s. Dès lors, il ne reste qu'un pas à franchir pour tomber dans l'impersonnalité la plus fade: "De plus, on a oublié ce que c'est que d'être un homme ".

Le souci de préserver la naïveté, l'originalité, la primitivité - ce que Kierkegaard admirait tant chez les Grecs et chez PoulMartin M $\theta$ ller - c'est refaçonner l'homme à sa propre image ; la vie humaine prise sur l'imagination (savoir) se déshumanise. L'imagination (Phantasie) doit forcément se comprendre comme pur savoir (ren Viden); ce pur savoir, et Kierkegaard insiste làdessus fortement, s'identifie à la "méthode historicisante " (den historiserende Methode). Tout ce qui vient après la "voie royale kantienne ", et plus spécialement ceux qui font des "abstractions", ceux-là sont perdus dans des "combinaisons insensées"?

Il convient donc de remédier à cet état " malhonnête " de la connaissance en proposant une communication assez forte et assez personnalisée qui puisse secouer l'inertie du "public" (Pu-

3. Pap. VIII, 2, B, 81 .

4. Pap. VIII, 2, B, 82, 2.

5. Pap. VIII, 2, B, 81, 2

6. Pap. VIII, 2, B, 81, 3

7. Pap. VIII, 2, B, 81, 8 
blikum) : "Toute communication de la vérité est devenue abstraite : le public est maintenant l'instance ; les feuilles s'appellent la rédaction; les professeurs la spéculation ; les pasteurs sont la méditattion ; personne, personne n'ose dire " je »! Mais puisque la première condition absolue de toute communication de la vérité est la personnalité, comment alors la vérité peut-elle trouver son compte à cette ventriloquerie! Il s'agissait donc de remettre la personnalité en place. Dans ces circonstances, commencer tout de go par son propre moi, quand le monde est si vicié à ne plus jamais entendre un " je ", c'était impossible. Ma tâche alors fut d'inventer des personnalités d'écrivain et de les faire surgir en pleine réalité de la vie, pour habituer tout de même un peu les hommes à suivre ce discours à la première personne. Mais le revirement de cette abstraction inhumaine vers la personnalité, c'est là ma tâche. " ${ }^{8}$

Après ce survol critique de l'état du savoir à l'époque de Kierkegaard, l'analyse passe à l'objet même de la communication. La problématique est double : expliquer la signification de la communication indirecte, en dégager sa validité pour toute communication relevant de l'esthétique, de l'éthique et du religieux; d'autre part, en tant que nouvelle forme d'expression, procéder à une détermination plus précise de ses rapports avec la communication directe. Nous traduisons le tableau dans son ensemble; il révèle l'essentiel de la doctrine kierkegaardienne du "ce qui doit être appris" (Læren om det at lære)"

"Dès que je pense à ce qui doit être communiqué, je pense à quatre choses : 1) l'objet ; 2 ) le destinateur (ou le communicateur, l'encodeur) ; 3) le destinataire (ou.le receveur, le décodeur) ; 4) la communication ${ }^{10}$.

\section{lère Distinction}

Quand c'est réfléchi sur l'objet

Objet

Communication de con- Communication d'habilité. naissance.

8. Pap. X, 1, A, 531.

9. Pap. V, C, 6 .

10. Pap. VIII, 2, B, 83, p. 158. Voir R. Jakobson : Essais de linguistique générale, p. 213.
Ce que cela signifie. L'absence d'objet montre que la réfle- 
xion, et donc également la distinction, va dans le sens de l'objet ou à partir de l'objet, (mais) précisément négative dans le sens de l'objet ou à partir de l'objet.

Division de l'habilité

Habilité esthétique.

Habilité éthique ou bien Devoir-habilité (aucun objet). Habilité religieuse ou bien Devoir-habilité (où il y a, en ce sens, un objet qui est en premier lieu une communication de connaissance).

\section{2e Distinction}

Quand c'est réfléchi sur la communication

A. "La communication " par rapport au " médium "

\section{Médium de l'imagination \\ Médium de la réalité}

Toute communication de connaissance est dans le médium de l'imagination.
La communication de l'habilité est dans le médium de la réalité - habilité esthétique (est) relative

- habilité éthique (est) absolue

- habilité religieuse est relative, en autant qu'elle soit ici une communication de connaissance.

B. Réfléchi sur la " communication"

Communication directe

Toute communication de connaissance est communication directe.
Communication indirecte bilité est communication indirecte :
Toute communication de l'ha- 
1) la communication d'habilité esthétique est communication directe, mais une communication directe d'habilité, donc une communication indirecte ; 2) la communication d'habilité éthique est absolument une communication indirecte ;

3) la communication d'habilité religieuse est, en ce sens, communication directe qui est, en premier lieu, une communication de connaissance, mais, en principe, une communication indirecte.

\section{3e Distinction}

Quand c'est réfléchi sur le

\section{Destinateur \\ Destinataire}

A. Réfléchi à la fois sur le destinateur et sur le destinataire : communication d'habilité esthétique.

B. Réfléchi primordialement sur le destinataire : communication d'habilité éthique (Maïeutique)

C. Réfléchi primordialement sur le destinateur : communication d'habilité religieuse (le destinateur a autorité en ce qui concerne la communication de la connaissance qui est ici en premier lieu)".

Maintes questions peuvent se poser quant au bien-fondé de cette "dialectique ", en grande partie inspirée des leçons de Tennemann sur Sextus-Empiricus ${ }^{12}$; toutefois, il ne fait pas de doute que la " dialectique de la communication", ainsi présentée et articulée, constitue un document de première importance pour la recherche kierkegaardienne, notamment sur les rapports de la communication indirecte avec la pseudonymie. Voyons de plus près les quatre structures constitutives de la "communication".

11. Pap. VIIII, 2, 84, p. $160^{\circ}$ :

12. Pap. IV A, 198, et V, C, 6 . 
1. L'objet: Gjenstanden :

La théorie de la communication kierkegaardienne pose de façon très nette l'opposition entre Viden (connaissance) et Kunnen (habilité)). La "Viden", c'est - qu'on se souvienne de l'introduction au Concept de l'Angoisse - la volonté de reconduire à son domaine propre toute science, tout savoir, toute connaissance afin d'éviter tout mélange d'une science, d'un savoir, d'une connaissance à l'autre. En gros, c'est la spéculation, le système, Hegel. La "Viden " désigne aussi, en tant que formant l'un des pôles de l'ensemble linguistique, les sentences (satser), propositions, etc . .., qui articulent le "discours" de toute science et fondent sa logicité.

La communication de la "Viden " s'établit entre destinateur et destinataire : le destinateur $s$ 'attache à vouloir rendre signifiant un fond conceptuel qu'il transmet au destinataire et que celui-ci doit commenter, interpréter, déchiffrer théoriquement.

La communication de la "Kunnen " (habilité), opposée à la communication de la "Viden", couvre un champ de "signification" encore plus vaste (esthétique, éthique, religion). Dans une communication de la "Kunnen", le déploiement signifiant concerne surtout le destinataire. En effet, celui-ci doit être tenté, doit pratiquer le message, bref, il doit, d'une certaine manière, réunir le pratique au théorique. C'est pourquoi Kierkegaard affirme que la communication de la "Viden " possède un objet (Gjenstand) (1ère distinction) déterminé (savoir, connaissance) alors que la communication de la "Kunnen" en est évidemment dépossédée. Kierkegaard donne l'exemple suivant : "Un exemple de la méprise à comprendre un enseignement d'habilité comme un enseignement de connaissance. Un sous-officier dit à une recrue : "Vous devez tenir les rangs ! "Recrue : "Oui, je le ferai ". Sousofficier: "Oui, de plus, vous ne devez pas parler à l'exercice". Recrue: "Ah! je ne peux pas parler! Bien, puisque vous le dites! "Sous-officier: "Nom de Dieu ! vous ne devez pas parler à l'exercice!" Recrue : "Oui, oui, ne vous fâchez pas, puisque je ne peux parler, je m'en abstiendrai à l'avenir. " ${ }^{13}$

Cet exemple un peu naiff illustre la confusion possible entre une communication de la "Viden" et une communication de la

13. Pap. VIII, 2, B, 81, 14, p. 149. 
"Kunnen": "L'objet de la communication n'est pas un savoir, mais une réalisation. " ${ }^{14}$ Mais par ailleurs, la communication de l'habilité esthétique (æthethisk Kunnen) peut se transformer en communication de savoir ; c'est le cas pour l'enseignant (Lærer) qui a atteint un savoir-faire (une habilité) proportionnel à son savoir (virtuosité, achèvement et excellence dans l'art en général). C'est là l'unique exception avec la connaissance de la religiosité- $A$.

L'éthique donc est essentiellement une communication de "Kunnen", et si elle est devenue une communication de savoir, c'est qu'on a perdu, avec l'abstraction systématique du siècle, le sens de l'éthique : enseigner l'éthique, la communiquer comme un savoir, c'est engager une communication de savoir qui ne peut être que contraire à l'éthique ${ }^{15}$. Certes, on sait par ailleurs que l'éthique pose des normes qui sont objectives et auxquelles l'individu doit se conformer. La difficulté vient du fait que l'objet d'une communication doit être un savoir ${ }^{16}$. Mais l'éthique est beaucoup plus une pratique qu'une objectivation. Kierkegaard se réfère à l'individu qui peut avoir une connaissance a priori sur ce qui est objectivement juste en éthique, - c'est même une présupposition compréhensive nécessaire, - mais cela est indifférent (ligegyldigt) comme il le mentionne lui-même. D'autant que les catégories du "destinateur" (Meddeler) et du "destinataire " (Modtager) s'évanouissent d'elles-mêmes dans une communication d'éthique: "Tout individu connaît l'éthique. Comment est modifiée la dialectique de la communication? 1) L'objet s'efface ; tout le monde le connaissant, il ne peut être communiqué - chercher de cette manière à communiquer l'éthique est a-éthique. 2) Le destinateur s'efface ; car puisque chacun le connaît, il s'ensuit que l'un ne peut pas le communiquer à l'autre. 3) Le destinataire s'efface; car si le destinateur s'efface, le destinataire s'efface aussi. " ${ }^{27}$

Comment Kierkegaard s'en sort-il ? La communication de l'éthique peut se passer du savoir, car, finalement, ce qui compte, c'est la réalisation; d'autre part, Kierkegaard remarque qu'il " n'y a qu'un destinateur: Dieu $»^{18}$.

14. Pap. VIII, 2, B, 81, 20, p. 150. + "Gjenstand er som viist, ikke en Viden, men en Kunst, en Realisation".

15. Pap. VIII, 2, B, 81, 5, p. 145.

16. Pap. VIII, 2, B, 81, 8, p. 146. "Gjenstanden maa være en Viden".

17. Pap. VIII, 2, B, 81, 11, p. 148.

18. Pap. VIII, 2, B, 8I, 11, p. 148. 
Sous cet angle, la coupure, entre une communication de "Viden" et de "Kunnen" ayant trait à l'éthique, ne semble pas du tout satisfaisante - du moins, pas au niveau de l'objet. Il faudra donc analyser ce problème dans l'axe de la communication elle-même.

Le religieux "subit " un traitement identique ; comme " 1 'homme comme tel ne connaît pas la religion - il doit y avoir au préalable une brève communication de savoir " ${ }^{19}$. Kierkegaard veut ici éviter toute confusion possible : "L'habilité religieuse : il y a ici un moment de savoir et en outre un objet. Mais ce n'est qu'en premier lieu. La communication n'est pas vraiment une communication de savoir, mais une communication d'habilité. Qu'il y ait un moment de savoir, cela est surtout valable en ce qui concerne le christianisme. Mais c'est seulement une communication provisoire $^{20}$. " C'est ainsi qu'avec les notions de "Viden» et de "Kunnen ", Kierkegaard jette les bases d'une théorie de la communication directe et indirecte.

Il y a communication directe lorsqu'il y a un objet (Gjenstand) de communication du destinateur au destinataire, à savoir : une connaissance que le destinateur communique au destinataire. L'objet du savoir, la communication de la scientificité, est une communication "indifférente". Une communication de savoir empirique a toujours lieu entre un destinateur et un destinataire ; l'accent toutefois est placé sur l'objet de la communication, non sur le destinateur ou sur le destinataire. Et toute communication "indifférente " est complètement désengagée de tout problème humain et personnel ${ }^{21}$.

\section{Le destinateur, Meddeler:}

Comme nous venons de le voir, une communication de savoir (Viden) est centrée exclusivement sur l'objet ; l'évolution, la trans-

19. Pap. VIII, 2, B, 82, 13, p. 157

20. Pap. VIII, 2, B, 85, 29, p. 167.

21. L'analyse recoupe certaines notions déjà pressenties par Johannes Climacus dans De omnitus : IV, b, 1., pp. 133-34-35-36., où l'on mentionne l'indifférence de la philosophie objective (celle qui commence par le doute) quant à la relation destinateur-destinataire. Voir Poul-Martin Moller (Tanker over Muligheden) et Sibbern. 
formation, etc..., n'ont aucun effet sur la communication ellemême. Elle doit, malgré tout, pouvoir compter sur une "prédisposition réceptive " (Modtageligheden) de la part du destinataire : "On présuppose une vérité chez le destinataire, une prédisposition réceptive, mais l'accent ne tombe pas primordialement sur ce qu'il a ; mais sur ce qu'il reçoit, l'objet. $»^{22}$ C'est d'autant plus vrai, reprend Kierkegaard, que la communication du savoir, par son insistance sur l'objet, conduit nécessairement à l'objectif, à l'impersonnel (det Upersonlige) ${ }^{23}$. La communication de savoir ne concerne - suivant le schéma kierkegaardien - ni la deuxième ni la troisième distinction.

La communication d'habilité (Kunnen) est fort différente de la communication de savoir en ce sens qu'elle mène vers la personnalité : "Partout où il y a réflexion sur la communication, le destinateur, le destinataire, c'est d'une manière ou d'une autre, une communication d'habilité et qui tend alors vers la personnalité. $2^{24}$ Dans une communication d'habilité éthique, le destinateur doit absolument être un " éthicien existant " (en existerende Ethiker) ${ }^{25}$.

De plus, vouloir communiquer l'éthique est au plus haut point "illusoire " (Bedrag), et le destinateur qui s'obstine à vouloir " communiquer " l'éthique est dépourvu de tout sérieux : " (...) la communication indirecte est, dans un premier temps, une illusion, justement parce que le désir même de communiquer l'éthique porte à illusionner. Dans la marge: cette illusion signifie avant tout que le destinateur n'est pas un homme sérieux, (. . .) $»^{26}$.

Le destinateur doit lui-même se confondre avec ce qu'il veut communiquer - c'est un " incitateur " qui invite à la réalisation. Quelles sont ses "obligations"?

En premier lieu, il " n'ose communiquer qu'indirectement : 1) parce qu'il doit exprimer qu'il n'est pas lui-même un enseignant, mais un enseigné ; 2) parce qu'il doit exprimer que le destinataire est au courant ; 3) parce que l'éthique est la tâche suivant laquelle l'individu est amené à se sentir seul devant le divin ${ }^{27}$.

\footnotetext{
22. Pap. VIII, 2, B, 85, 2, p. 161.

23. Pap. VIII, 2, B, 84, p. 160.

24. Pap. VIII, 2, B, 84, p. 160.

25. Pap. VIII, 2, B, 81, 5, p. 145

26. Pap. VIII, 2, B, 81, 22, p. 151

27. Pap. VIII, 2, B, 81, 20, p. 150 .
} 
$\grave{A}$ ces obligations s'ajoutent deux " axiomes " exceptionnellement dialectiques: "Le dialectique du fait que le destinateur doit se contraindre lui-même ${ }^{28}$ et "Le dialectique du fait que le destinateur doit avoir des yeux derrière la tête, mettant en place l'acquisition réelle de la communication $"^{29}$.

Le destinateur éthicien, à la fois éthicien existant et passé maître (dans l'acceptation kierkegaardienne) dans l'art de la maïeutique, prend au sérieux le "tu dois " (Du skal) : "Dès lors que l'accent doit absolument tomber sur le "tu dois", il ne peut donc pas être question de communication de savoir $"^{30}$. En ce sens, le destinateur doit faire sentir que la communication en cours c'est-à-dire l'enseignement communiqué (Underviisning) — est essentiellement éducative (Opdragelse), contrairement à une communication strictement éthique (au sens d'art, Kunst) qui est surtout orientée vers l'adresse, l'excellence, l'achèvement (Färdighed) ${ }^{31}$.

Le destinateur religieux, pour sa part, doit faire siennes les remarques qui ont trait au destinateur éthicien : être existentiel et faire de la maïeutique Cela dit, il lui faut se mettre à l'école du savoir (Viden), d'une manière provisoire (forel $\phi$ big), il va sans dire, et puis, ensuite, passer à la communication de l'habilité (Kunnen) et s'égaliser ainsi avec l'éthicien : " La différence entre une éducation éthique et une éducation éthico-religieuse réside uniquement en ceci : que l'éducation éthique n'est rien d'autre que l'humain (et n'a rien d'autre que d'humain) tandis que l'éducation religieuse doit, en un premier temps, communiquer un savoir. L'homme connaît l'éthique, mais chrétiennement parlant, l'homme ne connaît pas le religieux, il doit y avoir au préalable une petite communication de savoir -- et alors se produit le même rapport que pour l'éthique. L'enseignement, la communication ne doivent pas être comme pour le savoir, mais une éducation, un exercice, un art-enseignement. $n^{32}$

Kierkegaard ajoute, ce qui ne manque pas d'intérêt : "C'est

\footnotetext{
28. Pap. VIII, 2, B, 81, 31, p. 152.

29. Pap. VIII, 2, B, 81, 32, p. 152.

30. Pap. VIII, 2, B, 85, 9, p. 162.

31. Pap. VIII, 2, B, 85, 9, p. 162.

32. Pap. VIII, 2, B, 82, 13, pp. 156-57.
} 
là mon mérite avec les pseudonymes : avoir découvert la mä̈eutique à travers le christianisme. " $"{ }^{33}$

\section{Le destinataire: Modtager:}

Dans l'aménagement dialectique de la communication kierkegaardienne, le destinataire se caractérise par son activité : "destinataire est un terme actif ", précise Kierkegaard, "nous n'avons pas de termes passifs ${ }^{34}$. Le rôle actif du destinataire s'établit en fonction du rôle que lui attribue le destinateur; ils forment un couple indissociable ${ }^{35}$.

Pour saisir la portée exacte de leur relation, référons-nous à la dialectique de la communication. Dans une communication de l'habilité (Kunnen) éthique, laquelle ne saurait en rien modifier l'intention personnelle, et par l'intermédiaire du destinateur, le destinataire doit être entraîné à la pratique des buts et des finalités éthiques. En d'autres termes, le destinataire, remplissant son rôle actif, est supposé participer d'une manière entière au dictum de l'éthique.

La différence entre une communication d'habilité éthique et une communication religieuse est beaucoup plus fonctionnelle que réelle. Dans un premier temps, la communication religieuse pose l'univocité destinateur-destinataire en insistant plus particulièrement sur l'aspect réception; l'association destinateurdestinataire, avec son insistance marquée sur le " recevoir ", révélera la capacité active du destinataire : le reçu doit rayonner à partir d'un maniement, d'une reconquête.

Dans un second temps, la communication religieuse se fusionne à la communication de l'habilité éthique : le destinataire est "seul avec l'aide d'un autre $"^{36}$. Et là se trouvent mises en évidence les remarques sur la maïeutique : cet art de reprise, de dévoilement est l'invitation la plus directe à se "prendre en main ", à

33. Pap. VIII, 2, B, 82, 13, p. 157.

34. Pap. VIII, 2, B, 81, 7, p. 146. "At Modtager er et activ Ord, at vi intet passivt Ord have"

35. Pap. VIII, 2, B, 81,11 , p. 148

36. Pap. VIII, 2, B, 84, p. 160. 
se réaliser. En ce sens, l'unité de ces deux types de communication signifie la coïncidence dynamique entre incitation et activité.

\section{La communication indirecte:}

La communication directe (Viden), contaminée par la "spéculation " et le savoir objectif, ne retient que fort peu l'attention de Kierkegaard. Il s'en sert pour mieux faire ressortir la communication indirecte, par contraste analytique, mais il est tout à fait évident qu'elle est, au plus haut degré, foncièrement inintéressante. La grande coupure entre communication directe et communication indirecte reflète les différences par rapport au médium d'expression utilisé : la communication directe (Viden) emprunte le médium de l'imagination (Phantasie-Mediet) alors que la communication indirecte (Kunnen) se sert du médium de la réalité (Virkeligheden). Dans ces notes sur la dialectique de la communication, Kierkegaard penche naturellement vers la communication indirecte, la seule qui lui paraisse utile pour toute communication relevant de l'esthétique et de l'éthico-religieux. Nous en donnons ici les principales articulations :

a) La communication indirecte est dépendante de son mode d'expression qui est le médium de la réalité : "La communication de l'éthique ne peut être donnée que dans la réalité, de telle sorte que le destinateur ou l'enseignant doit exister en elle, et dans la situation de la réalité. " ${ }^{37}$ Dans une communication par le médium de la réalité, le côté théorique de la communication du savoir est totalement délaissé au profit de l'aspect pratique (Praxis) ; on sait, entre autres, qu'une communication de savoir n'a qu'un intérêt fugace, minimal, pour ce qui est de la relation entre destinateur et destinataire. Il en est bien autrement pour une communication indirecte d'habilité qui insiste, avec raison, sur la mise en œuvre et sur le caractère intime et liant du destinateur et du destinataire, avec une règle particulière pour le destinateur qui doit " se connaître et s'efforcer d'être continuellement ce qu'il communique ${ }^{38}$.

b) La communication indirecte peut prêter à de graves équivoques - l'éthique par exemple : "Toute communication indi-

37. Pap. VIII, 2, B, 28, p. 152.

38. Pap. VIII, 2, B, 81, 27, p. 152. 
recte est différente de la directe en ceci que la communication indirecte est, en premier lieu, une illusion, précisément parce que vouloir communiquer l'éthique porte à illusionner. "199 Puisqu'il n'existe pas de communication de savoir à ce niveau-ci, le destinateur n'a pas à communiquer de savoir au destinataire, mais s'il le faisait, il démontrerait qu'il n'est pas encore parvenu à une complète "finition" (Færdighed), à un achèvement significatif de luimême. Le destinateur, on le sait maintenant, doit lui-même mettre en pratique les préceptes éthiques, et c'est par lui que la communication indirecte doit agir sur le destinataire et l'amener au contrôle et à la maîtrise de lui-même.

Ce qui veut dire : le contenu de l'éthique, du religieux (aussi l'esthétique) doit être réalisé à la fois par le destinateur et par le destinataire, reconnaissant ainsi le droit de la communication indirecte (Kunnen) à revendiquer d'être la communication par excellence. 